\title{
The spatio-temporal dynamics of deviance and target detection in the passive and active auditory oddball paradigm: a sLORETA study
}

\author{
Christoph Justen ${ }^{1,2}$ and Cornelia Herbert ${ }^{2 *}$
}

\begin{abstract}
Background: Numerous studies have investigated the neural underpinnings of passive and active deviance and target detection in the well-known auditory oddball paradigm by means of event-related potentials (ERPs) or functional magnetic resonance imaging (fMRI). The present auditory oddball study investigates the spatio-temporal dynamics of passive versus active deviance and target detection by analyzing amplitude modulations of early and late ERPs while at the same time exploring the neural sources underling this modulation with standardized low-resolution brain electromagnetic tomography (sLORETA).
\end{abstract}

Methods: A 64-channel EEG was recorded from twelve healthy right-handed participants while listening to'standards'and 'deviants' (500 vs. $1000 \mathrm{~Hz}$ pure tones) during a passive (block 1) and an active (block 2) listening condition. During passive listening, participants had to simply listen to the tones. During active listening they had to attend and press a key in response to the deviant tones.

Results: Passive and active listening elicited an N1 component, a mismatch negativity (MMN) as difference potential (whose amplitudes were temporally overlapping with the N1) and a P3 component. N1/MMN and P3 amplitudes were significantly more pronounced for deviants as compared to standards during both listening conditions. Active listening augmented P3 modulation to deviants significantly compared to passive listening, whereas deviance detection as indexed by N1/MMN modulation was unaffected by the task. During passive listening, SLORETA contrasts (deviants > standards) revealed significant activations in the right superior temporal gyrus (STG) and the lingual gyri bilaterally (N1/MMN) as well as in the left and right insulae (P3). During active listening, significant activations were found for the N1/MMN in the right inferior parietal lobule (IPL) and for the P3 in multiple cortical regions (e.g., precuneus).

Discussion: The results provide evidence for the hypothesis that passive as well as active deviance and target detection elicit cortical activations in spatially distributed brain regions and neural networks including the ventral attention network (VAN), dorsal attention network (DAN) and salience network (SN). Based on the temporal activation of the neural sources underlying ERP modulations, a neurophysiological model of passive and active deviance and target detection is proposed which can be tested in future studies.

Keywords: EEG, Source localization, N1, Mismatch negativity, MMN, P3, Attention, Salience, Attention networks

\footnotetext{
*Correspondence: cornelia.herbert@uni-ulm.de

${ }^{2}$ Institute of Psychology and Education, Applied Emotion and Motivation

Research, University of UIm, Ulm, Germany

Full list of author information is available at the end of the article
} 


\section{Background}

Rapid orientation of selective attention towards changes in the acoustic environment is essential for successful interaction with our environment. Remarkably, the deviance of a perceived stimulus determines to what extent attention is captured by this stimulus. In other words, the more deviant a stimulus is, the more attention is allocated towards this particular stimulus [1]. In the auditory domain, the essential ability of deviance detection has been studied mostly in the so-called oddball paradigm [2]. In this experimental paradigm two auditory stimuli (e.g., pure tones) are presented as targets (deviants) or as non-targets (standards). Deviants are usually embedded in a continuous stream of standards and differ from standards in at least one perceptual dimension (for instance, a difference in frequency, pitch or loudness). This difference in stimulus presentation frequency and in physical stimulus properties between deviants and standards seems sufficient to prioritize processing of deviants over standards during passive and hence, involuntary (bottom-up) stimulus processing. In addition, detection of deviant stimuli may benefit from active, i.e., voluntary (top-down) controlled stimulus processing, for instance, when deviants are actively attended by the participants as targets for task-related voluntary discrimination of deviants and standards [3].

Due to its simplicity, the auditory oddball paradigm constitutes an ideal research paradigm for cognitive neuroscience to investigate the neural mechanisms of auditory deviance and target detection during passive and active listening conditions. So far, several event-related potential (ERP) and functional magnetic resonance imaging (fMRI) studies have been conducted to determine on the one hand the time course and on the other hand the brain regions underlying auditory deviance and target detection in this paradigm (for meta-analytic research e.g., [4]). Effects have been investigated during task conditions of passive or active listening (for ERP studies see e.g., [5-8]; for fMRI see e.g., [9-13]). In general, this body of research provided important insight into auditory processing under unattended (passive) and attended (active) task conditions while at the same time raising questions about the temporal activation of specific brain regions and brain networks involved in auditory deviance and target processing during passive versus active listening conditions.

According to influential theoretical models of attention allocation proposed by Corbetta and Shulman [14] and data from a recent meta-analysis based on functional imaging studies [4], processing of deviant stimuli (in contrast to standard stimuli) is associated with bottom-up as well as top-down stimulus processing and activation of two distinct fronto-parietal networks, namely the 'dorsal attention network' (DAN) and the 'ventral attention network' (VAN) [4, 14, 15]. The DAN comprises the superior parietal lobule/precuneus (SPL; Brodmann area (BA7)), the intraparietal sulcus (IPS; BA 6/7), parts of the middle temporal cortex (BA 21) and the inferior frontal junction (IFJ; BA 6/9/44). The VAN comprises the temporoparietal junction (TPJ; BAs 39/40), the supramarginal gyrus (SMG; BA 39), the superior temporal gyrus (STG; BAs 22/41/42), the frontal operculum (FO; BA 44/45/47), the inferior frontal gyrus (IFG; BAs 44/45/47), the anterior cingulate cortex (ACC; BAs 24/32/33) and the anterior insula (AI, BA 13) $[4,14,15]$. According to recent meta-analytic research, putting together the results of 75 neuroimaging studies using either auditory or visual oddball paradigms, processing of deviants versus standards activates the DAN and the VAN differently during passive versus active stimulus processing [4]. Parts of the DAN seem to be activated during the processing of deviants and standards, and specifically its frontal parts seem to be involved in voluntary target detection in line with the idea of the dorsolateral prefrontal cortex (DLPFC; BAs $8 / 9 / 10 / 46$ ) playing a central role in top-down selective attentional control $[4,16]$. In contrast to the DAN, the VAN seems to be exclusively involved in the detection of deviant stimuli and brain regions belonging to the VAN seem to be more active when deviants are voluntarily attended as compared to conditions in which they are not attended (e.g., during passive listening). The VAN is therefore supposed to enable 'attentional shifting' to deviant stimuli, probably to initiate an appropriate behavioral response when deviants are important for the task [17].

However, this raises questions about which brain networks and brain regions might be involved in auditory deviance processing when there is no task at hand. Theoretically, some brain structures of the VAN such as the insula and ACC but also brain regions of the DAN (e.g., precuneus) form part of the 'salience network' (SN) [18, 19]. Amongst these brain regions in particular the insular cortex is considered to be involved in bottom-up detection of salient events and the selection of these for additional processing [20]. In addition, it has been shown that in the oddball paradigm, attentional processing (e.g., attention orientation) to auditory stimuli can activate visual processing regions (e.g., lateral and medial occipital areas) and hence, processing regions typically engaged in object recognition and in the perception of visual objects $[21,22]$ in tasks requiring spatial attention (e.g., see [23]). Thus, specific brain regions in the visual cortex (such as the lateral occipital cortex (LOC) or the lingual gyrus (LG) in the medial visual cortex) may also be activated during attentive processing of acoustic stimuli [24]. However, whether the aforementioned visual processing areas 
are also activated during task conditions of passive and thus, unattended listening to deviant and standard pure tones in the oddball paradigm needs to be investigated further [25-27].

So far, the above outlined assumptions about the activation of the DAN and VAN during auditory processing have been derived mainly from meta-analytic research [4] including functional neuroimaging studies. Thus, it has not been investigated whether activation of the DAN and VAN could be modeled by source imaging techniques that rely on EEG activity such as SLORETA. Crucially, exploring the time course of auditory deviance and target detection through electroencephalography (EEG) and event-related brain potentials (ERPs) and simultaneous targeting the brain regions of the VAN and DAN by means of sLORETA could be especially fruitful in situations where fMRI is not available or too costly. Moreover, due to its high temporal resolution in the millisecond time-range, EEG and EEG based source imaging offers the possibility to investigate the time course of deviance and target detection in the auditory oddball paradigm (e.g., be investigating modulation of ERPs) while at the same time the neural sources underlying ERP modulation can be estimated within the same temporal resolution of milliseconds. Akin to functional imaging, in EEG-ERP source imaging studies, effects can be investigated during passive listening conditions, during which intentional discrimination between deviants and standards via explicit instructions is not required. In addition, investigation of effects associated with active listening conditions (e.g., explicit instruction to attend to deviant stimuli) is also possible, thus allowing direct comparisons of ERPs, their neural sources and their modulation during deviance and target detection in a within-subject design including task conditions of passive and active listening.

Regarding the time course of auditory deviance and target detection, ERP components most consistently elicited during the auditory oddball paradigm are the N1, the N2a or auditory mismatch negativity (N2a/MMN; e.g., see [28]), and the late P3 (e.g., see [29-31]).Whereas the $\mathrm{N} 1$ and MMN are brain potentials whose amplitudes are significantly influenced by differences in physical stimulus properties, the magnitude of the P3 amplitude is significantly influenced by cognitive and task demands (e.g., see [32]). The N1 and the MMN have been found during passive listening as well as during active processing of deviant stimuli [33]. In contrast to the N1, the MMN is computed by subtracting the averaged ERP waveform of standard stimuli from the averaged ERP waveform of deviant stimuli. The resulting negative deflection is peaking between 100 and $250 \mathrm{~ms}$ after stimulus onset [34]; amplitudes of the MMN being often more pronounced at fronto-central electrode sites [35]. Regarding its latency, the MMN can overlap with the auditory N1 component (e.g., see [36]) peaking between 80 and $120 \mathrm{~ms}$ poststimulus [37], especially if deviant and standard stimuli are clearly perceptually distinct from each other. The auditory MMN has been proposed to indicate mostly pre-attentive sensory stimulus discrimination [38] as well as automatic, and thus involuntary auditory change detection [39, 40]. Hence, the modulation of the MMN is assumed to be driven by involuntary mechanisms of the brain's sensory processing system matching the incoming stimulus to its internally stored representation (or template). This matching is considered to occur "unconsciously" and temporally prior to stimulus categorization [41].

Amplitudes of the P3, temporally following early brain potentials such as the $\mathrm{N} 1$ and $\mathrm{N} 2 \mathrm{a} / \mathrm{MMN}$, are most pronounced between 300 and $450 \mathrm{~ms}$ after stimulus-onset at central-parietal as well as parietal electrode sites [30]. Previous auditory oddball studies suggest that the P3 is elicited only by sufficiently deviant stimuli [42] with P3 amplitudes being larger in response to voluntarily attended than unattended deviant stimuli (e.g., see [5]). Accordingly, the P3 is thought to reflect voluntary switch of attention [39, 40,43] and in depth-processing of a stimulus signaling stimulus evaluation based on memory [44] and context updating $[29,45]$.

Taken together, one might expect N1, N2a/MMN and P3 modulation to be differentially sensitive to task effects. Moreover, one could speculate that different neural sources may underlie N1, MMN and P3 modulation during deviance and target detection in passive and active listening conditions. Compared to the huge amount of EEG-ERP studies or fMRI studies investigating either the time course or the brain regions involved in auditory deviant and target detection only few studies investigated both, the time course and the neural sources of auditory deviance and target detection in the oddball paradigm, for instance with combined EEG-fMRI methodology [13]). Although combining fMRI and EEG/ERP methodology is beneficial, localization of neural generators of ERP components might still be affected by the relatively poor temporal resolution of conventional functional neuroimaging techniques [46]. To this end, (standardized) low-resolution brain electromagnetic tomography (LORETA and sLORETA, respectively) have been developed to make assumptions about the location of neural generators of brain electrical activity derived from multi-channel EEG recordings with high temporal resolution [47-49]. The validity of (s)LORETA has been confirmed in a number of combined fMRI-EEG studies (e.g., see [48]). More specifically, Mulert et al. compared brain activations in an active auditory oddball paradigm as measured by fMRI with brain activations derived from 
EEG and LORETA [50]. The comparison between fMRI and LORETA activation patterns contrasting deviants versus standards during active and hence, attended processing of deviants versus standards showed concurrent activations in brain regions associated with the VAN, namely the TPJ (BAs 39/40), the supplementary motor area (SMA; BA 6), the ACC (BAs 24/32/33), the middle frontal gyrus (MFG; BA 46) and the anterior insula (AI, BA 13). Interestingly, neural generators of the $P 3$ partially overlapping with the VAN, including TPJ (BAs 39/40), DLPFC (BAs 8/9/10/46), ACC (BAs 24/32/33) and parts of the parietal and temporal cortices could be already confirmed in recent EEG-sLORETA studies (e.g., see [50-52]). Thus, source localization with sLORETA can be a means to successfully estimate neural sources underlying target detection in the oddball paradigm without losing information about the time course.

\section{Aim and objectives of the present study}

Building upon the previous findings outlined above, the aims of the present auditory oddball study were to methodologically combine EEG-ERP methodology with sLORETA in the oddball task in order to (a) investigate the time course of auditory deviant and target detection by studying ERP modulation in active as well as in passive listening conditions and (b) examine the temporal activation of brain regions contributing to the observed ERP modulation patterns in the two different listening conditions. Thus, by taking advantage of the high temporal resolution of the sLORETA source imaging technique the present study allows to explore which brain regions belonging to the hypothesized brain networks (i.e., DAN, VAN and SN) might contribute to the modulation of early and late ERP components elicited by deviant and standard pure tones during active and passive listening. In line with previous research and the assumptions outlined above the present study aimed at testing the following hypotheses and open questions regarding the temporal activation of specific brain regions during auditory deviant and target detection: firstly, we were interested in whether passive listening of deviant compared to standard stimuli will be associated with activations in brain regions belonging to the VAN or the $\mathrm{SN}$, indicating involuntary attentional orientation towards deviant stimuli when no behavioral response is required. Secondly, we were interested in whether during passive listening brain regions belonging to the VAN and, or the SN will contribute as neural sources to automatic deviant detection (MMN) or to later processing stages of deviance processing as indicated by modulation of the P3. Thirdly, during the attended oddball paradigm, activations in brain regions associated with the VAN and the DAN could be expected in the time window of the MMN and the P3 component indicating (1) involuntary attentional orientation towards deviant stimuli and (2) voluntary modulation of attention in order to maintain attentional resources for responding behaviorally to deviant stimuli. Finally, previous fMRI studies found activation in the visual cortex during passive auditory processing in the oddball paradigm. Thus, cortical activations in lateral $/$ medial occipital areas could also be expected to occur in the present study during passive listening and activation of these brain regions might be associated with the modulation of early and late ERPs.

\section{Methods \\ Participants}

Twelve right-handed university students ( 7 females, 5 males) aged between 19 and 26 years $(M=21.3$ years, $S D=2.15)$ participated in the present study. All participants were in good health and reported no psychological or hearing disorders. The experiment (including EEG recordings) was conducted at the Institute of Psychology of the German Sport University Cologne, Germany and was part of a larger project of the authors (see funding sources, and [53]). The experimental protocol complied with the Declaration of Helsinki and was approved by the local ethics committee of the German Sport University Cologne, Germany. All participants gave written informed consent prior to the start of the experiment and received a momentary compensation for their participation.

\section{General procedure}

Prior to the start of the EEG session, participants had been seated in a comfortable chair and were informed about the general procedures of the experiment including EEG recordings. The experiment consisted of a passive and active auditory oddball paradigm; the passive condition (block 1) being always followed by the active condition (block 2). Block order was kept constant across participants as not to confound passive with active processing due to possible carry over effects. Particularly, the induction of an overt (behavioral) response to deviant pure tones during the active paradigm and hence active orientation of attention towards the deviant stimuli may have influenced deviance processing during passive listening if the active paradigm had been presented first (cf., [5]). Auditory stimuli were presented at constant sound pressure level of about $75 \mathrm{~dB} / \mathrm{SPL}$ using Shure SHR440 on-ear headphones (Shure, Niles, IL, USA). During the passive oddball paradigm (block 1), participants were instructed to listen passively to the presented auditory stimuli. Accordingly, no behavioral response was required. For the active oddball paradigm (block 2), participants were told to respond to the deviant pure tones 
as quickly as possible by pressing the space bar on a keyboard with their right index finger. To avoid horizontal eye movements (saccades) while listening, participants were instructed to fixate their view on a fixation cross presented on the video screen. Additionally, participants were told to keep their eyes open during stimulus presentations (block 1 and block 2, respectively).

\section{Passive and active tone oddball paradigm}

The oddball paradigm consisted of two auditory stimuli, a low $(500 \mathrm{~Hz})$ pure tone which was presented as "standard" and a high $(1000 \mathrm{~Hz})$ pure tone which was presented as "deviant" stimulus (cf., [54]). Thus, both pure tones differed only in the frequency domain and both were perceptually sufficiently different from each other to be clearly recognized as standard and deviant during stimulus presentation. Both auditory stimuli had durations of $50 \mathrm{~ms}$ (including a $5 \mathrm{~ms}$ fade-in/out time). The experimental paradigm consisted of in total of 400 trials (325 standard trials and 75 deviant trials, respectively) with a fixed inter-stimulus-interval (ISI) of $950 \mathrm{~ms}$ duration. Stimulus presentation and recording of responses were controlled by the Inquisit 4.0 software package (Millisecond Software, Seattle, WA, USA). The experimental script was downloaded from the official Inquisit website ([55]).

\section{EEG recordings}

Continuous EEG data (sampling frequency: $2.048 \mathrm{~Hz}$ ) were recorded from $64 \mathrm{Ag} / \mathrm{AgCl}$ sintered electrode using standardized EEG recording sites $(\mathrm{Fp} 1, \mathrm{Fpz}, \mathrm{Fp} 2$, AF7, AF3, AF4, AF8, F7, F5, F3, F1, Fz, F2, F4, F6, F8, FT7, FC5, FC3, FC1, FCz, FC2, FC4, FC6, FT8, T7, C5, C3, C1, Cz, C2, C4, C6, T8, TP7, CP5, CP3, CP1, CPz, CP2, CP4, CP6, TP8, P7, P5, P3, P1, Pz, P2, P4, P6, P8, PO7, PO5, PO3, POz, PO4, PO6, PO8, O1, Oz, O2, M1 and M2). Electrodes were mounted on a Waveguard EEG cap (Advanced Neuro Technology B.V., Enschede, The Netherlands). The electrode sites of this montage were arranged according to the international 10/10-system [56] and all EEG channels were referenced to the common average of all scalp electrodes. Forehead electrode $\mathrm{AFz}$ was used as ground electrode. Blue Sensor N disc electrodes (Ambu, Ballerup, Denmark) were placed at the outer canthi of both eyes and additionally below the left eye for horizontal and vertical electrooculography (HEOG and VEOG, respectively). All electrode impedances were kept below $10 \mathrm{k} \Omega$.

\section{Preprocessing of EEG data}

EEG data were preprocessed offline with the ASALAB (Version: 4.7.8) software package [57]. Preprocessing included down-sampling to $512 \mathrm{~Hz}$, band-pass filtering between 0.5 and $20 \mathrm{~Hz}(24 \mathrm{~dB} /$ oct) and bandstop (notch) filtering of $50 \mathrm{~Hz}(24 \mathrm{~dB} / \mathrm{oct})$. Eye blinks and saccade-related artifacts were corrected with an artifact correction feature based on principle component analysis (PCA), as introduced by [58]. Further data analysis of artifact-free EEG data involved re-referencing to linked mastoids/linked ears (M1 and M2), segmentation into epochs for each stimulus type and condition, resulting in epochs from $100 \mathrm{~ms}$ before and $700 \mathrm{~ms}$ after stimulus onset for each of the four different experimental conditions including "Deviants" and "Standards" during the passive listening condition as well as "Deviants" and "Standards" during the active listening condition. All epochs were scanned for artifacts using an automated artifact detection algorithm. The automatic artifact rejection threshold in all epochs (between -100 and $700 \mathrm{~ms}$ after stimulus onset) was set to $\pm 100 \mu \mathrm{V}$ within a $400 \mathrm{~ms}$ interval (between 0 and $400 \mathrm{~ms}$ after stimulus onset). Epochs exceeding this threshold were discarded. All extracted epochs were baseline corrected using the interval from $100 \mathrm{~ms}$ before stimulus onset. Referencing, segmentation and baseline correction as well as any further analysis step (e.g., averaging) were done in EEGLAB (Version: 13.4.4b; [59]) and MATLAB (Version: R2013a, 8.1.0.604; The MathWorks Inc., Natick, MA, USA). Grand averaged ERPs were generated for all four aforementioned experimental conditions.

\section{ERP analysis and statistics}

Detection of the time windows in which ERP amplitudes including the $\mathrm{N} 1$ and $\mathrm{P} 3$ were most pronounced was done with the built-in EEGLAB function "statcond" [60]. Averaged ERPs to deviant and standard stimuli (during both passive and active listening condition, respectively) were submitted to non-parametric paired $t$-tests with 5.000 permutations at all time points between 0 (stimulus onset) and $700 \mathrm{~ms}$ after stimulus onset with 62 electrode sites included. To control for multiple comparisons, the false discovery rate (FDR; for an introduction, see [61]) was used for all statistical analyses (as implemented in the EEGLAB function "FDR") with an FDR-level of 5\% $(q=0.05)$. By maintaining reasonable limits on the likelihood of false discoveries (i.e., it is suitable for a reasonable correction on a large number of comparisons), the FDR procedure provides a much better spatial and temporal resolution as compared to parametric $t$-tests using the "classical" Bonferroni correction (for an introduction, see [62]).

Regarding analysis of the MMN and the P3, difference waves were calculated from the averaged ERP waveforms elicited by deviant and standard pure tones in both experimental conditions ("Deviants" and "Standards" during the passive listening condition and "Deviants" and 
"Standards" during the active listening condition, respectively) using the Maas Univariate ERP Toolbox [63-65]. All time points in the MMN (50-150 ms) and the P3 time window (200-450 ms) as well all 62 scalp electrodes were included in the statistical tests (i.e., 3.224 and 9.610 total comparisons, respectively).

Difference waves were submitted to a repeated measures, two-tailed cluster-based permutation test based on the cluster mass statistic [66] using a family-wise alpha level of 0.05 . Repeated measures $t$-tests were performed for each contrast ("Deviants" and "Standards" during the passive listening condition and "Deviants" and "Standards" during the active listening condition, respectively) using the original data and 2.500 random within-participant permutations of the data. For each permutation, all $t$-scores corresponding to uncorrected $p$-values of 0.01 or less were clustered: to this end, electrodes within a distance of approximately $5.44 \mathrm{~cm}$ were considered spatial neighbors and adjacent time points were considered temporal neighbors. The sum of the $t$-scores in each cluster is the "mass" of that cluster and the most extreme cluster mass in each of the 2.501 sets of tests was used to estimate the distribution of the null hypothesis (i.e., no difference between "Deviants" and "Standards" in the passive as well as "Deviants" and "Standards" in the active listening condition, respectively). More specifically, the assumption of the null hypothesis of the permutation test is that positive differences between conditions could have just as likely been negative differences and vice versa. Thus, the distribution of the null hypothesis is symmetric around a difference of 0 . The permutation cluster mass percentile ranking of each cluster from the observed data was used to derive its $p$-value. The $p$-value of the cluster was assigned to each member of the cluster and $t$-scores that were not included in a cluster were given a $p$-value of 1 . This permutation test analysis was used instead of the conventional testing of mean amplitude values because it provides much better spatial and temporal resolution while maintaining control of the family-wise alpha level (i.e., it corrects for a large number of comparisons). 2.500 permutations were used to estimate the distribution of the null hypothesis as it is over twice the number recommend by [67] for a family-wise alpha level of 0.01 . For the cluster mass permutation test, all desired $p$-values, critical $t$-scores, and the corresponding family-wise alpha levels are reported (please see Results, "Passive tone oddball paradigm-ERPs (N1, MMN and P3)" and "Active tone oddball paradigm-ERPs (N1, MMN and P3)" sections).

\section{EEG source localization analysis}

Neural generators of the ERPs were analyzed with the sLORETA software (University Hospital of Psychiatry, Zürich, Switzerland; [68]). Source estimations were done on single participants' data and were restricted to the time windows showing significant differences in the ERP waveforms between the contrasted conditions (see EEGERP results, "Behavioral results-active tone oddball paradigm", "Passive tone oddball paradigm-ERPs (N1, MMN and P3)", "Active tone oddball paradigm-ERPs (N1, MMN and P3)", "Passive and active deviants-passive and active standards ERPs" sections). In SLORETA, computations are based on a realistic head model [69] using the MNI-152 template [70], with the three-dimensional solution space restricted to cortical gray matter, as determined by the probabilistic Talairach atlas [71, 72]. The standard electrode positions of the MNI-152 template were taken from [56] as well as [73]. The intracerebral volume is partitioned in 6.239 voxels with a spatial resolution of $5 \times 5 \times 5 \mathrm{~mm}$ each. Thus, the obtained sLORETA images represent the standardized electric activity at each voxel in the neuroanatomic Montreal Neurological Institute (MNI) space as the exact magnitude of the estimated current density. Anatomical labels are reported in Brodmann areas (BA) in line with MNI space, with a correction to Talairach space [74]. For sLORETA no preregistration of individual subjects is required. The matching or co-registration of the individual EEG data with the MNI-152 template is based on the scalp-recorded electric potential distribution and computed on the basis of the cortical three-dimensional distribution of current density. Thus, the software automatically co-registers the data according to the head surface points (electrode locations provided in the electrode configuration file). As introduced by Nichols and Holmes [75], statistical nonparametric mapping (SnPM) was used to compute the standardized intracerebral current density distribution at time intervals or time points showing significant differences based on non-parametric voxel-by-voxel paired samples $t$-tests (with 5.000 permutations) on the threedimensional sLORETA images. Statistical significance was assessed by defining critical thresholds $\left(t_{\text {crit }}\right)$ corrected for multiple comparisons $(p<0.01$ and $p<0.05$, respectively) for all tested voxels and time windows. The null hypotheses equaled the assumption that there were no differences between "Deviants" and "Standards" in both the passive and active listening condition, respectively.

Standardized current density values at each voxel have been computed in the solution space as a linear and weighted sum of the scalp electric potentials. Activation of a given voxel was based on the smoothness assumption, meaning that neighboring voxels show a highly synchronous activity [76]. Support comes from electrophysiological studies showing that electrical activity of neighboring neural populations is highly correlated [76, 77]. As proposed by [78], activated voxels exceeding $t_{\text {crit }}$ 
were considered as being regions of cortical activation. Finally, statistical analysis resulted in a three-dimensional intracerebral current density distribution and obtained cortical regions were classified in relation to their corresponding BA [79] and normalized coordinates (Talairach and $\mathrm{MMI}$, respectively).

\section{Results}

Behavioral results - active tone oddball paradigm

Participants' reaction times (RTs) to deviant pure tones were between 223 and $379 \mathrm{~ms}(M=285.6 \mathrm{~ms}, S D=38.5)$. On average, participants responded to all trials with deviant pure tones with high accuracy $(M=74.8$, $S D=0.7)$.

\section{Passive tone oddball paradigm-ERPs (N1, MMN and P3)}

Passive listening to deviant and standard pure tones elicited an N1 component as well as a P300 component. As shown in Fig. 1 and as revealed by the grand average of the ERP waveforms, N1 amplitudes were most pronounced between 67 and $129 \mathrm{~ms}$ (peak at about $95 \mathrm{~ms}$ ) after stimulus onset; P3 amplitudes were most pronounced during 232-354 ms (peak at about $300 \mathrm{~ms}$ ) post-stimulus. In addition, difference waves-subtracting

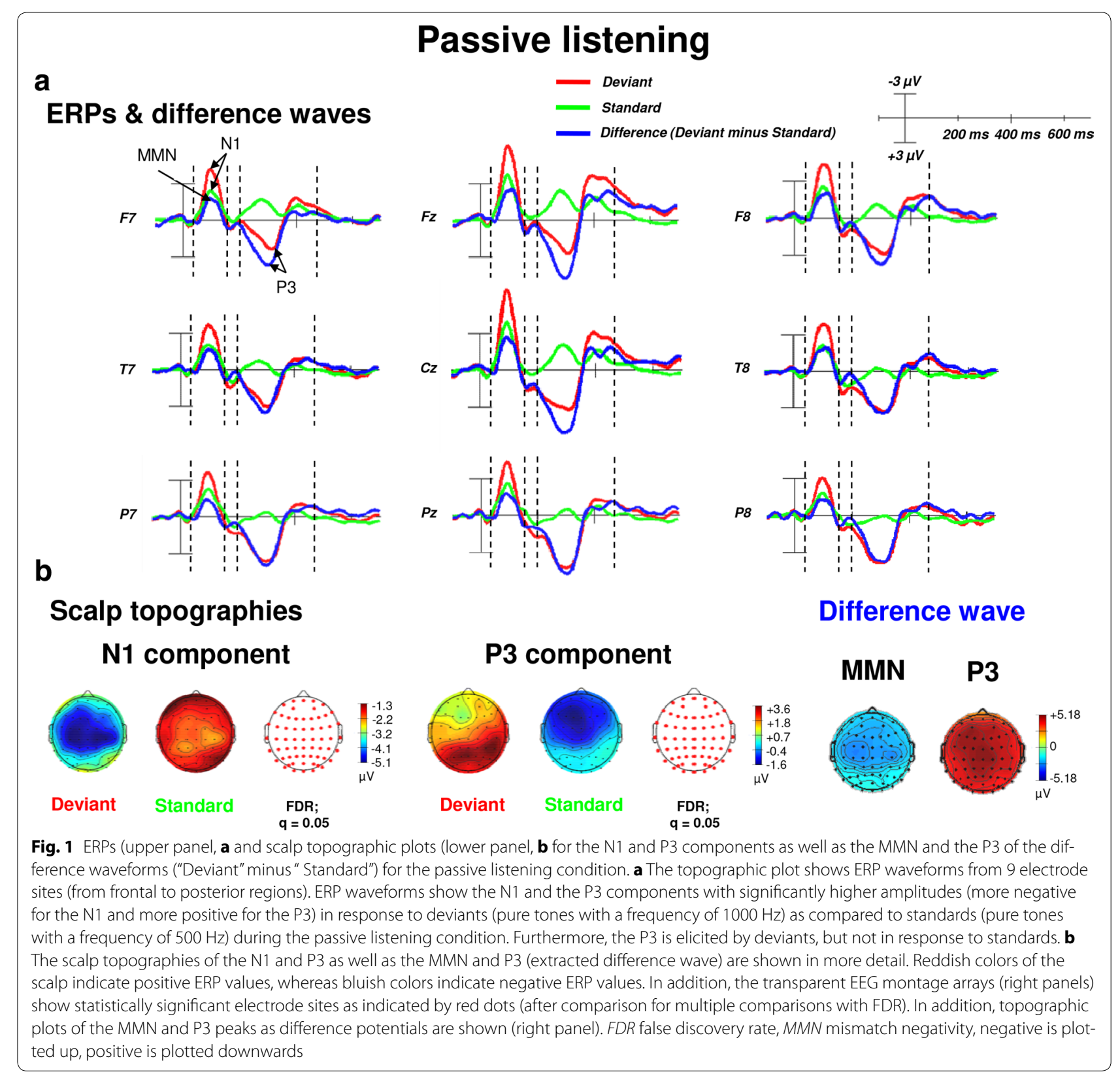


ERP waveforms elicited in response to "Deviants" from "Standards"-during the passive listening condition revealed an MMN component. As shown in Fig. 1, the amplitude of the MMN overlapped with the time window of the N1 component between 66 and $128 \mathrm{~ms}$ after stimulus onset. As also shown in Fig. 1, amplitudes of the N1, MMN and the P3 were significantly more pronounced for deviants as compared to standards.

Regarding early time windows, the maximum number of statistically significant differences between "Deviants" and "Standards" occurred in the N1/MMN time window between 82 and $103 \mathrm{~ms}$ post-stimulus (desired $p=.05$, critical $t$-score $=-2.20$ corresponding to a family-wise alpha-level of .05 and a Bonferroni test-wise alpha-level of .000004; see also "Passive tone oddball paradigm" section; electrodes: anterior-frontal and fronto-temporal: AF7, AF4, AF3, FT8, Fp1, Fp2, Fpz; frontal: F8, F7, F6, F5, F4, F3, F2, F1, Fz, FC6, FC5, FC4, FC3, FC2, FC1, FCz; central: C6, C4, C3, C2, C1, Cz; temporal: T8). This seems to be in line with previous findings of an 'early' MMN peaking at about $100 \mathrm{~ms}$ [32]. To ensure the validity of this interpretation, electrodes were re-referenced offline to a common average reference (CAR). According to the literature, CAR or a nose reference are recommended as these montages are known to be the best reference sites to robustly determine the MMN [80]. As expected, this procedure confirmed the characteristic polarity inversion of the extracted MMN at both mastoid electrodes sites (M1 and M2, respectively). Thus, the extracted MMN of the difference wave occurred in the averaged time window of the $\mathrm{N} 1$ component observed in the averaged ERP waveforms [36].

For the P3 component the maximum number of statistically significant differences between "Deviants" and "Standards" was observed between 269 and $322 \mathrm{~ms}$ post-stimulus (desired $p=.05$, critical $t$-score $=2.20$ corresponding to a family-wise alpha-level of .05 and a Bonferroni test-wise alpha-level of .000008; see also "Passive tone oddball paradigm" section; electrodes: frontal: AF8, AF7, AF4, AF3, Fp1, Fp2, Fpz, F8, F7, F6, F5, F4, F3, F2, F1, Fz, FC6, FC5, FC4, FC3, FC2, FC1, FCz, FT8, FT7; central: C6, C5, C4, C3, C2, C1, Cz, CP6, CP5, CP4, CP3, CP2, CP1, CPz; temporal: T8, T7, TP8, TP7; parietal: P8, P7, P6, P5, P4, P3, P2, P1, Pz, PO8, PO7, PO6, PO5, PO4, PO3, POz; occipital: O2, O1, Oz).

\section{Active tone oddball paradigm-ERPs (N1, MMN and P3)}

Active listening to deviant and standard pure tones elicited an N1, an MMN as well as a P300 component. As shown in Fig. 2, the MMN component (obtained from the difference wave by subtracting "Deviants" from "Standards" during the active listening condition) was again overlapping with the time window of the N1 component elicited in response to "Deviants" and "Standards". N1 amplitudes were most pronounced in the time window from 60 to $114 \mathrm{~ms}$ (peak: at about $90 \mathrm{~ms}$ ), the MMN amplitude was most pronounced between 56 and 117 ms post-stimulus and the P3 amplitudes were most pronounced during $232-378 \mathrm{~ms}$ (peak: at about $300 \mathrm{~ms}$ ) post-stimulus. As also shown in Fig. 2, amplitudes of the N1, MMN and the P3 were more pronounced for deviants as compared to standards, i.e., amplitudes were more negative going for the $\mathrm{N} 1$ and $\mathrm{MMN}$ and more positive going for the P3 when listening to "Deviants" as compared to "Standards".

Regarding early time windows (N1, MMN), the maximum number of statistically significant differences between "Deviants" 3.5.2and "Standards" was observed between 83 and $95 \mathrm{~ms}$ post-stimulus (desired $p=.05$, critical $t$-score $=-2.21$ corresponding to a family-wise alpha-level of .05 and a Bonferroni test-wise alpha-level of .000016; see also "Active tone oddball paradigm" section; electrodes: frontal: AF8, F8, F7, F6, FC6, FC5, FC4, FC3, FC2, FC1; central: C6, C5, C4, C3,C2, C1, Cz, CP6, CP4, CP3, CP2, CP1, CPz; parietal: Pz). Again (see "Passive tone oddball paradigm-ERPs (N1, MMN and P3)" section), re-referencing to CAR confirmed the characteristic polarity inversion of the extracted MMN at the left and right mastoid electrodes sites (M1 and M2, respectively).

For the P3 component the maximum number of statistically significant differences between "Deviants" and "Standards" was observed between 253 and $351 \mathrm{~ms}$ after stimulus onset (desired $p=.05$, critical $t$-score $=2.10$ corresponding to a family-wise alpha-level of .05 and a Bonferroni test-wise alpha-level of .000008; see also "Active tone oddball paradigm"; electrodes: frontal: AF8, AF7, AF4, AF3, Fp2, Fp1, Fpz, F8, F7, F6, F5, F4, F3, F2, F1, Fz, FC6, FC5, FC4, FC3, FC2, FC1, FCz, FT8, FT7; central: C6, C5, C4, C3, Cz, CP6, CP5, CP4, CP3, CP2, CP1, CPz, C6, C5, C4, C3, C2, C1, Cz, CP4, CP3, CPz; temporal: T8, T7, TP8, TP7; parietal: P8, P7, P6, P5, P4, P3, P2, P1, Pz, PO8, PO7, PO6, PO5, PO4, PO3, POz; occipital: O2, O1, $\mathrm{Oz})$.

\section{Passive and active deviants-passive and active standards ERPs}

Contrasting ERP waveforms elicited by passive and active deviant pure tones during both oddball listening conditions revealed significant differences in ERP amplitudes between 228 and $456 \mathrm{~ms}$ post-stimulus onset, corresponding to the P3 component (see also "Passive and active deviants" section). In this time window, the amplitudes were more negative going during passive as compared to active listening (see Figs. 1 and 2). No amplitude differences were found in earlier time windows. 


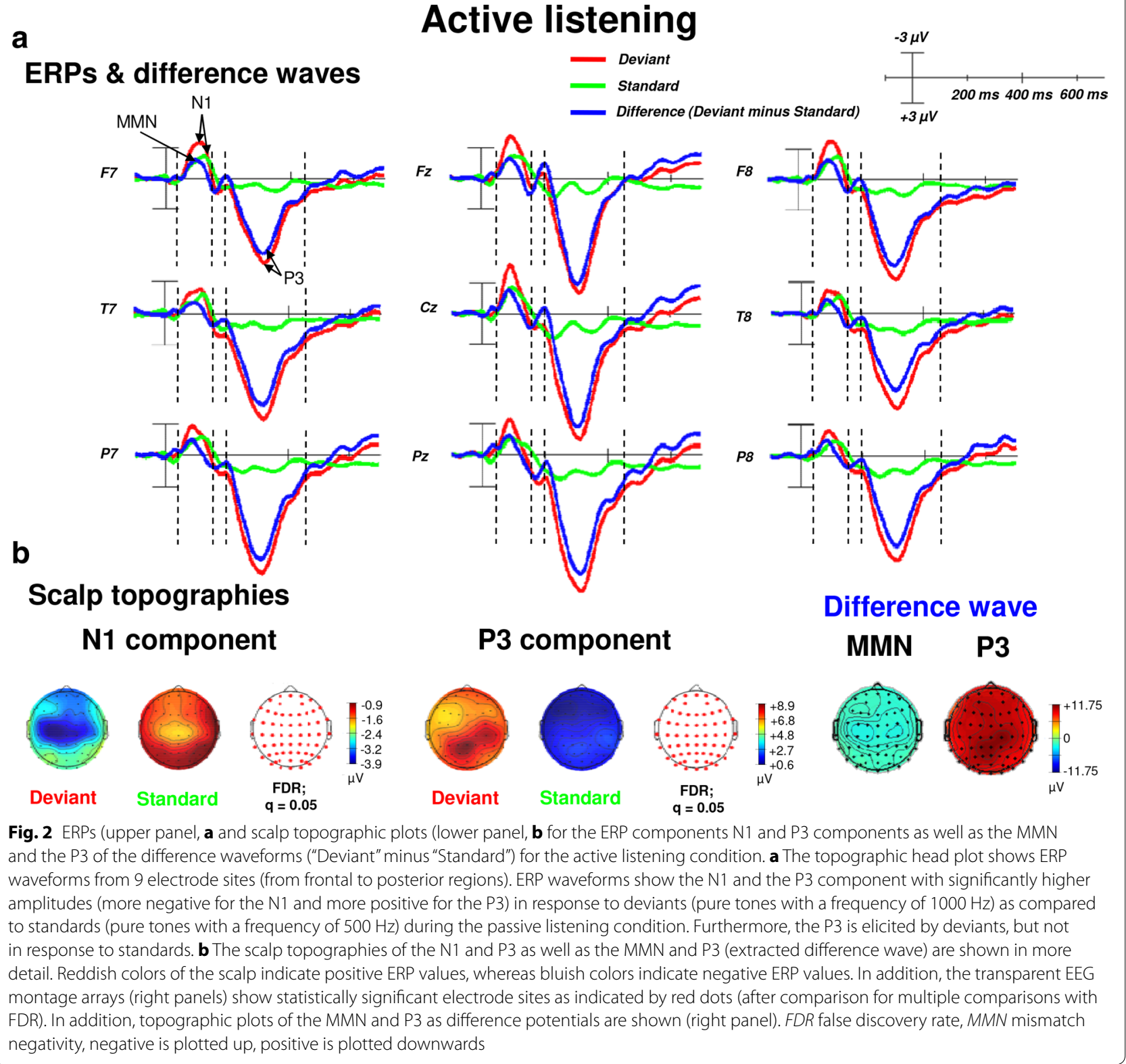

Contrasting ERP waveforms elicited by standard pure tones during active versus passive listening revealed a significant difference in ERP amplitudes between 190 and $418 \mathrm{~ms}$ after stimulus onset (see also "Passive and active standards" section). No significant differences were found in earlier time windows corresponding to the N1 or MMN component.

\section{sLORETA source localization analysis Passive tone oddball paradigm}

As shown in Fig. 3, contrasting deviant against standard pure tones with sLORETA during the passive oddball listening condition (contrast: "Deviants"> Standards") revealed significant activations in the lingual gyri (bilaterally) (BAs $17 / 18 / 19 ; t$-score $4.06 ; p<0.01$ ) as well as in the right superior temporal gyrus (STG; BAs 13/22/39/42; $t$-score $3.97 ; p<0.05$ ) between 82 and $103 \mathrm{~ms}$ after stimulus onset, which corresponds with the ERP analysis time window in which $\mathrm{N} 1$ and MMN amplitude differences were most pronounced, see "Passive tone oddball paradigm-ERPs (N1, MMN and P3)").

In addition, between 269 and $322 \mathrm{~ms}$ (P3 component) significant electrocortical activations included the insular cortex bilaterally (BA $13 ; t$-score $4.80 ; p<0.01$ ) and the right lingual gyrus (LG; BA 18 ; $t$-score $4.78 ; p<0.01$ ), see Fig. 3. For a complete overview of all retrieved 

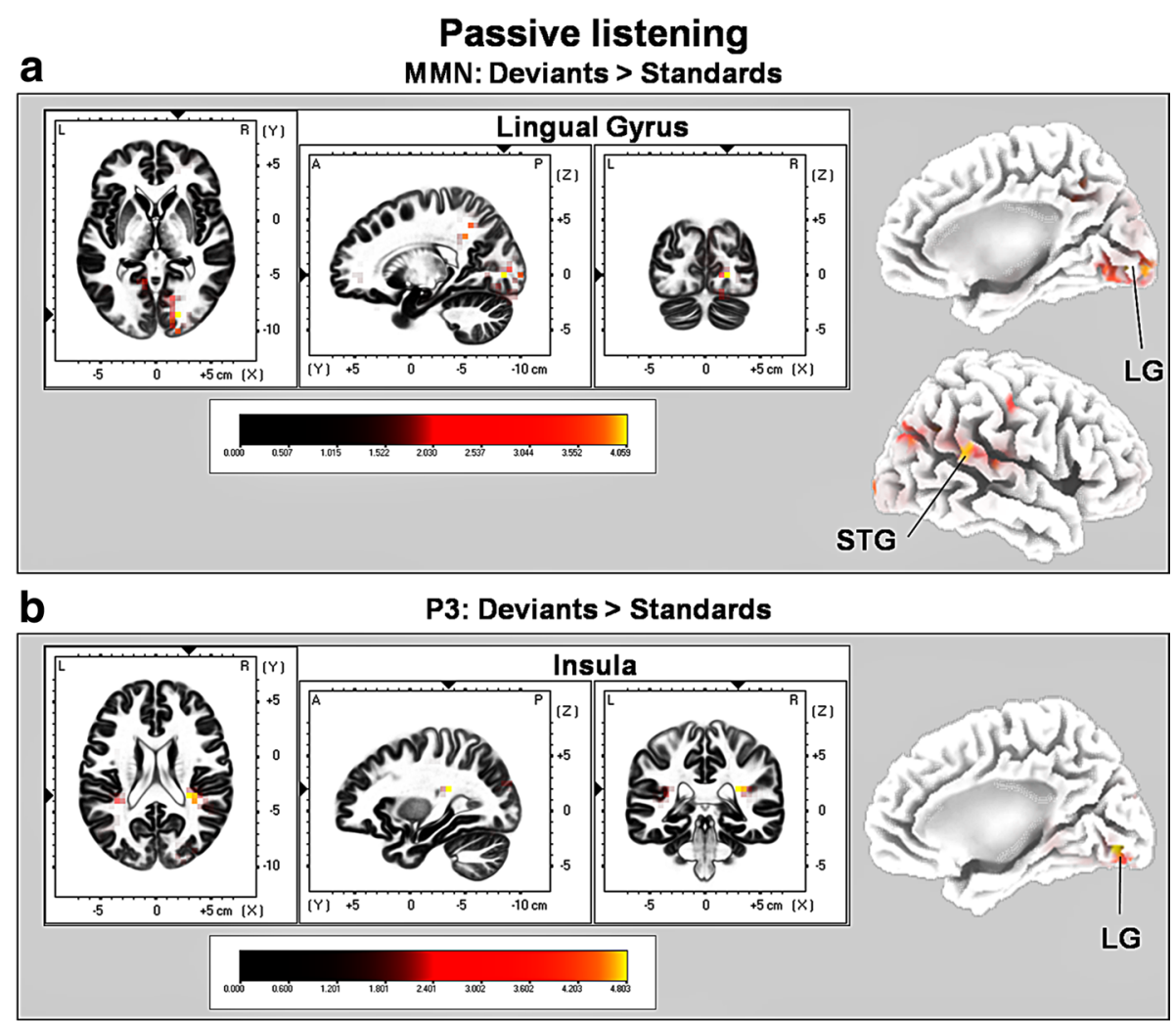

Active listening

C MMN: Deviants > Standards

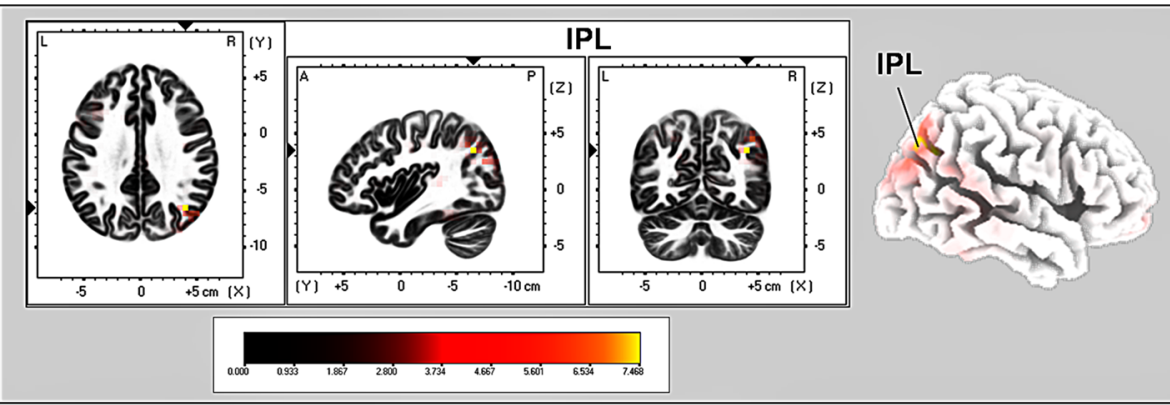

d

P3: Deviants > Standards
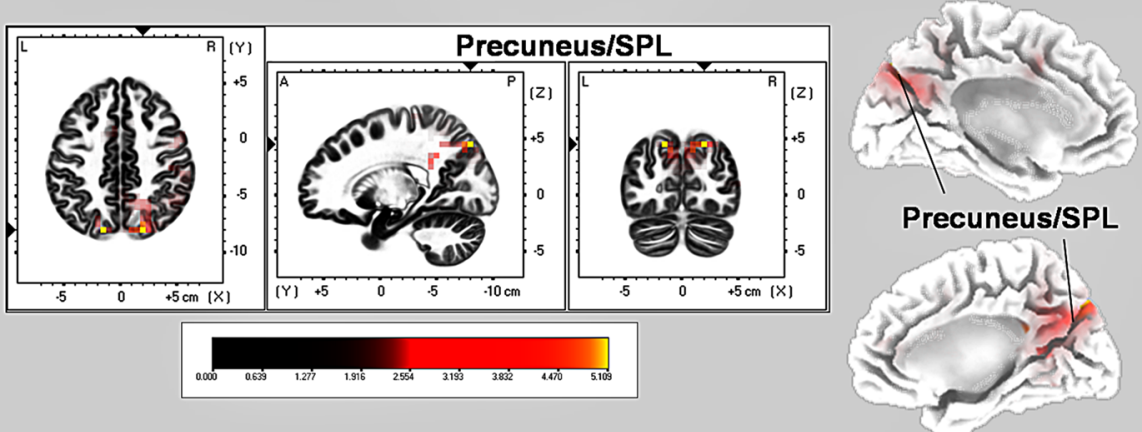
(See figure on previous page.)

Fig. 3 Results of the standardized low-resolution brain electrotomography (sLORETA) source localization analysis in the 'passive' and 'active' pure tone oddball paradigm. Images have been obtained after statistical non-parametric mapping (SnPM) and co-registration to the stereotaxic Talairach space based on the Co-Planar Stereotaxic Atlas of the Human Brain [72] and the probabilistic MNI-152 template [70]. Activated voxels are indicated by yellowish and reddish colors [after correction for multiple comparisons ( $p<0.01$ and $p<0.05$, respectively)]. a In the averaged time windows of the 'unattended' MMN component (80-123 ms), the peak of highest cortical activity has been found in the right LG (BAs 17/18/19) and right STG (BAs 13/22/39/42). b In the averaged time windows of the 'attended'MMN component (83-95 ms), the peak of highest cortical activity has been found in the right IPL (BAs 7/39/40). $\mathbf{c}$ In the averaged time windows of the 'unattended' $\mathrm{P} 3$ component (269-322 ms), the peak of highest cortical activity has been found in both insulae bilaterally (BA 13) and the right $L G$ (BA 18). $\mathbf{d}$ In the averaged time windows of the 'attended'P3 component (253-351 ms), the peak of highest cortical activity has been found in the precuneus/SPL bilaterally (BAs 7/19/23/21). $L$ left, $R$ right, $L G$ lingual gyrus, STG superior temporal gyrus, IPL inferior parietal lobule, SPL superior parietal lobule, MNI Montreal Neurological Institute, X, Y, Z corresponding MNI coordinates, BA Brodmann area

statistically significant results including all anatomical regions and activated voxels, see Tables 1 and 2 .

\section{Active tone oddball paradigm}

Contrasting deviant against standard pure tones in the active oddball listening condition (contrast: "Deviants" > "Standards") with sLORETA revealed significant activation in the right inferior parietal lobule (IPL; BAs $7 / 39 / 40 ; t$-score $=8.12 ; p<0.01$ ) between 83 and $95 \mathrm{~ms}$ after stimulus onset and hence, in the time window of the N1 and MMN amplitude (see ERP results in 3.3). Furthermore, significant activations were found in the precuneus bilaterally (BAs $7 / 19 / 23 / 31 ; t$-score 5.11 ; $p<0.01$ ), the cingulate cortices bilaterally (BA $31 ; t$-score $4.86 ; p>0.01)$, the superior temporal gyri bilaterally (STG; BAs $13 / 22 / 39 / 41 ; t$-score $4.79 ; p<0.01$ ), the left and right precentral gyri (BAs $4 / 6$; $t$-score $4.81 ; p<0.01$ ), the left and right postcentral gyri (BAs $2 / 3 / 7 / 40 ; t$-score 4.64; $p<0.01$ ), the right posterior cingulate cortex (BAs $23 / 30 / 31 ; t$-score $4.59, p<0.01)$ and the right intraparietal lobe (IPL, BAs 39/40; $t$-score 4.04; $p<0.01$ ) in the time window between 253 and $351 \mathrm{~ms}$ (overlapping with the time window of the P3 component), see Fig. 3.

For a complete overview of all retrieved statistically significant results including all anatomical regions and activated voxels, see Tables 3 and 4 .

\section{Passive and active deviants}

Contrasting "Deviants" elicited during active listening against "Deviants" elicited during passive listening (i.e., Deviants active $>$ Deviants passive) revealed significant differences in activation in the right middle frontal gyrus (MTG; BAs 6/8/9/10/46; $t$-score 5.24, $p<0.01$ ), the left precuneus (BAs $7 / 19 ; t$-score $5.06, p<0.01$ ), the right

Table 1 sLORETA results from the contrast: "Deviant" versus "Standard" (1000 vs. $500 \mathrm{~Hz}$ pure tones) in the N1/MMN time window from 80 to $123 \mathrm{~ms}$ post stimulus-onset during passive listening

\begin{tabular}{|c|c|c|c|c|c|c|c|c|c|c|c|c|}
\hline \multicolumn{4}{|l|}{ Brain region } & \multicolumn{6}{|c|}{ Coordinates (X, Y, Z) } & \multicolumn{2}{|c|}{$t$-value } & \multirow[t]{2}{*}{ No. of voxels } \\
\hline Structure & $\mathrm{BA}$ & Hemisphere & Lobe & \multicolumn{3}{|c|}{ Talairach (max.) } & \multicolumn{3}{|c|}{ MNI (max.) } & \multirow{2}{*}{$\frac{\text { Max. }}{4.06^{* *}}$} & \multirow{2}{*}{$\frac{\text { Min. }}{3.30^{*}}$} & \\
\hline Lingual gyrus & $17,18,19$ & $R / L$ & Occipital & 20 & -82 & 4 & 20 & -85 & 0 & & & 30 \\
\hline Superior Temporal Gyrus (STG) & $13,22,39,42$ & $\mathrm{R}$ & Temporal & 64 & 38 & 20 & 65 & -40 & 20 & $3.97^{*}$ & $3.29 *$ & 9 \\
\hline
\end{tabular}

Talairach/MNI coordinates and $t$-values are referred to the peak activity in each brain region. Italic numbers indicate maximal brain electrical activity in the corresponding BA. Only clusters of size $\geq 9$ voxels are reported

${ }^{* *} p<0.01,{ }^{*} p<0.05, L$ left, $R$ right, $B A$ Brodmann area, MNI Montreal Neurological Institute

Table 2 sLORETA results from the contrast: “Deviant" versus "Standard" (1000 vs. $500 \mathrm{~Hz}$ pure tones) in the N1/MMN time window from 83 to 95 ms post stimulus-onset during active listening

\begin{tabular}{|c|c|c|c|c|c|c|c|c|c|c|c|c|}
\hline \multicolumn{4}{|l|}{ Brain region } & \multicolumn{6}{|c|}{ Coordinates (X, Y, Z) } & \multicolumn{2}{|c|}{$t$-value } & \multirow[t]{2}{*}{ No. of voxels } \\
\hline Structure & $\mathrm{BA}$ & Hemisphere & Lobe & Tala & $\operatorname{rach}(r$ & ax.) & MN & (max.) & & Max. & Min. & \\
\hline Inferior Parietal Lobule (IPL) & $7,39,40$ & $\mathrm{R}$ & Parietal/Temporal & 45 & -66 & 40 & 45 & -70 & 40 & $8.12^{* *}$ & $4.66^{* *}$ & 10 \\
\hline
\end{tabular}

Talairach/MNI coordinates and $t$-values are referred to the peak activity in each brain region. Italic numbers indicate maximal brain electrical activity in the corresponding BA. Only clusters of size $\geq 9$ voxels are reported

${ }^{* *} p<0.01, L$ left, $R$ right, $B A$ Brodmann area, MNI Montreal Neurological Institute 
Table 3 sLORETA results from the contrast: "Deviant" versus "Standard" (1000 vs. $500 \mathrm{~Hz}$ pure tones) in the P3 time window from 269 to $322 \mathrm{~ms}$ post stimulus-onset during passive listening

\begin{tabular}{|c|c|c|c|c|c|c|c|c|c|c|c|c|}
\hline \multicolumn{4}{|l|}{ Brain region } & \multicolumn{6}{|c|}{ Coordinates $(X, Y, Z)$} & \multicolumn{2}{|c|}{$t$-value } & \multirow[t]{2}{*}{ No. of voxels } \\
\hline Structure & BA & Hemisphere & Lobe & Tal & $\mathrm{ch}(\mathrm{m}$ & & MN & nax.) & & Max. & Min. & \\
\hline Insula & 13 & $R / L$ & Sub-lobar & 30 & -33 & 20 & 30 & -35 & 20 & $4.80^{* *}$ & $3.59^{*}$ & 11 \\
\hline Lingual Gyrus & 18 & $\mathrm{R}$ & Occipital & 10 & -78 & 0 & 10 & -80 & -5 & $4.78^{* *}$ & $3.59^{*}$ & 11 \\
\hline
\end{tabular}

Talairach/MNI coordinates and $t$-values are referred to the peak activity in each brain region. Italic numbers indicate maximal brain electrical activity in the corresponding BA. Only clusters of size $\geq 9$ voxels are reported

** $p<0.01,{ }^{*} p<0.05, L$ left, $R$ right, $B A$ Brodmann area, MNI Montreal Neurological Institute

Table 4 sLORETA results from the contrast: "Deviant" versus "Standard" (1000 vs. $500 \mathrm{~Hz}$ pure tones) in the P3 time window from 253 to 351 ms post stimulus-onset during active listening

\begin{tabular}{|c|c|c|c|c|c|c|c|c|c|c|c|c|}
\hline \multicolumn{4}{|l|}{ Brain region } & \multicolumn{6}{|c|}{ Coordinates (X, Y, Z) } & \multicolumn{2}{|c|}{$t$-value } & \multirow[t]{2}{*}{ No. of voxels } \\
\hline \multirow{2}{*}{$\begin{array}{l}\text { Structure } \\
\text { Precuneus }\end{array}$} & \multirow{2}{*}{$\begin{array}{l}\text { BA } \\
7,19,23,31\end{array}$} & \multirow{2}{*}{$\begin{array}{l}\text { Hemisphere } \\
\text { R/L }\end{array}$} & \multirow{2}{*}{$\begin{array}{l}\text { Lobe } \\
\text { Parietal/Occipital }\end{array}$} & \multicolumn{3}{|c|}{ Talairach (max.) } & \multicolumn{3}{|c|}{ MNI (max.) } & \multirow{2}{*}{$\frac{\text { Max. }}{5.11^{* *}}$} & \multirow{2}{*}{$\frac{\text { Min. }}{4.03^{* *}}$} & \\
\hline & & & & 20 & -75 & 45 & 20 & -80 & 45 & & & 132 \\
\hline Cingulate Gyrus & 31 & $R / L$ & Limbic & 15 & -42 & 25 & 15 & -45 & 25 & $4.86^{* *}$ & $4.06^{* *}$ & 20 \\
\hline Precentral Gyrus & 4,6 & $\mathrm{R}$ & Frontal & 45 & -8 & 37 & 45 & -10 & 40 & $4.81^{* *}$ & $4.08^{* *}$ & 13 \\
\hline Superior Temporal Gyrus (STG) & $13,22,39,41$ & $R / L$ & Temporal & -50 & -28 & 15 & -50 & -30 & 15 & $4.79^{* *}$ & $4.04^{* *}$ & 9 \\
\hline Postcentral Gyrus & $2,3,7,40$ & $R / L$ & Parietal & -50 & -24 & 15 & -50 & -25 & 15 & $4.64^{* *}$ & $4.03^{* *}$ & 11 \\
\hline Cuneus & $7,18,19,30$ & $R / L$ & Occipital & -10 & -76 & 36 & -10 & -80 & 35 & $4.62^{* *}$ & $4.04^{* *}$ & 35 \\
\hline Posterior Cingulate & $23,30,31$ & $\mathrm{R}$ & Limbic & 10 & -53 & 16 & 10 & -55 & 15 & $4.59^{* *}$ & $4.05^{* *}$ & 13 \\
\hline Inferior Parietal Lobule (IPL) & 39,40 & $\mathrm{R}$ & Parietal & 35 & -47 & 39 & 35 & -50 & 40 & $4.45^{* *}$ & $4.04^{* *}$ & 22 \\
\hline
\end{tabular}

Talairach/MNI coordinates and $t$-values are referred to the peak activity in each brain region. Italic numbers indicate maximal brain electrical activity in the corresponding BA. Only clusters of size $\geq 9$ voxels are reported

${ }^{* *} p<0.01, L$ left, $R$ right, $B A$ Brodmann area, MNI Montreal Neurological Institute

inferior frontal gyrus (IFG; BAs 9/13/45; $t$-score 4.86 , $p<0.01$ ), the left postcentral gyrus (BAs $2 / 5 / 7 / 40 ; t$-score $4.80, p<0.01$ ), the left superior parietal lobule (SPL; BA 7; $t$-score 4.77, $p<0.01$ ), the right precentral gyrus (BAs $6 / 9 ; t$-score $4.36, p<0.01$ ), and the right inferior parietal lobule (IPL; BA 40; $t$-score 4.27, $p<0.01$ ) in time window between 244 and $343 \mathrm{~ms}$, corresponding to the time window in which amplitudes of the P3 component were most pronounced during passive and active listening conditions (see "Active tone oddball paradigm-ERPs (N1, MMN and P3)", "Passive and active deviants-passive and active standards ERPs" sections), see Table 5.

Table 5 sLORETA results from the contrast: "Deviant" during active listening versus "Deviant" during passive listening in the time window between 228 and 456 ms after stimulus onset

\begin{tabular}{|c|c|c|c|c|c|c|c|c|c|c|c|c|}
\hline \multicolumn{4}{|l|}{ Brain region } & \multicolumn{6}{|c|}{ Coordinates (X, Y, Z) } & \multicolumn{2}{|c|}{$t$-value } & \multirow[t]{2}{*}{ No. of voxels } \\
\hline \multirow{2}{*}{$\begin{array}{l}\text { Structure } \\
\text { Middle frontal gyrus }\end{array}$} & \multirow{2}{*}{$\begin{array}{l}\text { BA } \\
6,8,9,10,46\end{array}$} & \multirow{2}{*}{$\begin{array}{l}\text { Hemisphere } \\
R\end{array}$} & \multirow{2}{*}{$\begin{array}{l}\text { Lobe } \\
\text { Frontal }\end{array}$} & \multicolumn{3}{|c|}{ Talairach (max.) } & \multicolumn{2}{|c|}{ MNI (max.) } & & \multirow{2}{*}{$\frac{\max .}{5.24^{* *}}$} & \multirow{2}{*}{$\frac{\min .}{4.00^{* *}}$} & \\
\hline & & & & 54 & 12 & 36 & 55 & 10 & 40 & & & 27 \\
\hline Precuneus & 7,19 & $L$ & Parietal & -10 & -55 & 63 & -10 & -60 & 65 & $5.06^{* *}$ & $3.81^{*}$ & 47 \\
\hline Inferior frontal gyrus & $9,13,45$ & $R / L$ & Frontal & 54 & 11 & 32 & 55 & 10 & 35 & $4.86^{* *}$ & $3.81^{*}$ & 16 \\
\hline Superior parietal lobule & 7 & $R / L$ & Parietal & -15 & -51 & 58 & -15 & -55 & 60 & $4.77^{* *}$ & $3.81^{*}$ & 11 \\
\hline Precentral gyrus & 6,9 & $\mathrm{R}$ & Frontal & 45 & 21 & 36 & 45 & 20 & 40 & $4.42^{* *}$ & $3.81^{*}$ & 9 \\
\hline Inferior parietal lobule & 40 & $\mathrm{R}$ & Parietal & 50 & -37 & 43 & 50 & -40 & 45 & $4.27^{* *}$ & $3.92 * *$ & 12 \\
\hline
\end{tabular}

Talairach/MNI coordinates and $t$-values are referred to the peak activity in each brain region. Itlaic numbers indicate maximal brain electrical activity in the corresponding BA. Only clusters of size $\geq 9$ voxels are reported

${ }^{* *} p<0.01,{ }^{*} p<0.05, L$ left, $R$ right, $B A$ Brodmann area, MNI Montreal Neurological Institute 


\section{Passive and active standards}

Contrasting standards during both listening conditions (contrast: "Standards" active listening condition > "Standards" passive listening condition) revealed significant activation in the right middle frontal gyrus (BAs 10/11; $40,53,-11 ; t$-score $4.39 ; p<0.05)$ in the time window between 190 and $418 \mathrm{~ms}$. As this contrast revealed only three significant voxels, no table is provided.

\section{Discussion}

The present study examined the spatio-temporal dynamics of auditory deviance and target detection in the auditory oddball paradigm by combining the advantages of the EEG-ERP methodology and the sLORETA source localization technique within the same experiment and subjects (i.e., within subject design). The design included both, passive as well as active listening conditions to specify and contrast the neural mechanisms underlying active and passive deviance and target detection. To this end, participants were instructed to listen (1) passively to pure tones without giving an overt behavioral response (experimental block 1) and (2) to listen to pure tones while being engaged in an active task (experimental block 2) which afforded to distinguish between the two presented pure tones by responding to the deviants by giving an overt behavioral response (button press).

\section{Time course of passive and active deviance and target detection}

Passive and active listening elicited an N1 and P3 component and additionally an MMN as difference potential when deviants were contrasted against standards. Amplitudes of the MMN which temporally overlapped with the amplitudes of the N1 were significantly more negative for deviant as compared to standard pure tones during passive and active listening conditions. This modulation pattern is in line with those reported in previous ERP studies using comparable auditory oddball paradigms with pure tones (e.g., see [81, 82]). Comparisons between deviants or standards during active versus passive listening (see "Passive and active deviants - passive and active standards ERPs", "Passive and active deviants" and Passive and active standards" sections) revealed no significant amplitude differences in the time window of the N1/MMN when passive and active listening conditions were compared against each other. Thus, voluntary guidance of selective attention to deviants may not facilitate deviance detection in early time windows of cortical stimulus processing (N1/ MMN) beyond passive listening. This finding supports the assumption of particularly the MMN reflecting pre-attentive sensory stimulus discrimination [38] and automatic (involuntarily) auditory change detection [39, 40], i.e., processes that cannot be influenced by task-related attentive processes. Regarding the overlap between amplitudes of the $\mathrm{N} 1$ and the MMN additional explanations for MMN modulation during auditory deviance processing have been proposed: as shown in Figs. 1 and 2, the time window of the N1 significantly overlapped with the MMN. In addition, N1 amplitudes were significantly reduced to standards during passive and active listening conditions. This may be explained by the observation that neurons reacting to standards only show a reduced electrical activity due to repeated stimulus presentation leading to habituation of these particular neurons. In contrast, neurons that fire in response to deviants show a much higher electrical activity. According to the literature, this phenomenon might be explained by the so-called refractoriness of certain neurons and thus by their selective sensitivity to different frequencies [36, 83, 84]. Hence, due to the amplitude overlap of the MMN and the N1, MMN modulation could also result from neural adaption in the auditory cortex [85]. However, whether this mechanism actually underlies N1/ MMN overlap has not been sufficiently clarified yet and requires further neurophysiological testing.

\section{Neural sources of passive and active deviance and target detection \\ Early time windows (N1/MMN)}

When passive listening to "Deviants" vs. "Standards" was compared, source localization with sLORETA revealed activation in the left and right occipital cortex as well as in the right superior temporal gyrus (STG; BA 22) in the N1/ MMN time window. Activation of the right STG included the auditory cortex (BA 42) and multisensory association areas (BAs 39/22). This is well in line with the idea of bottom-up and stimulus-driven deviance detection. During active processing of "Deviants" vs. "Standards" the largest voxel cluster was located in the right inferior parietal lobule (IPL; BAs 39/40). The right IPL is part of the ventral attention network (VAN) and plays an important role in visuospatial attention and attentive monitoring of stimuli for goal-directed eye or limb movements [86]. Thus, during active listening IPL activation during early stages of deviance processing (i.e., in the N1/MMN time window) may be a consequence of anticipatory control of attention in order to maintain current task goals (i.e., the voluntary selection of deviant stimuli). Altogether, this suggests that during passive and active listening early stages of deviance processing may be modulated by different brain regions and neural processes although, at a cortical level, with respect to ERPs, $\mathrm{N} 1$ and MMN amplitude modulation did not differ significantly between passive and active listening conditions.

\section{Late time windows (P3)}

For the time window corresponding to the P3 component the contrast "Deviants" > "Standards" revealed activation 
of the right and left insula during passive listening. Activation of the left and the right insula in the P3 window during passive listening is in line with previous studies reporting involvement of the insula (in particular the right insula) in auditory processing [87] and target detection (e.g., see [88]). More specifically, the insula is part of the VAN and also part of the so-called salience network $(\mathrm{SN})[18,19]$ that marks stimulus events as salient for additional processing and mediates activation of and between brain networks involved in bottom-up and topdown controlled attention. Crucially, during active listening, P3 modulation elicited by deviants as compared to standards was associated with activation in a distributed network including the precuneus and surrounding areas in the superior parietal lobule (SPL; BA 7), the posterior cingulate cortex (PPC; BAs 23/31) as well as motorrelated areas. Most of the aforementioned brain regions form part of the dorsal attention networks (DAN). The present results therefore suggest that activation of brain regions belonging to the DAN network may occur only during active listening and during later time windows (P3) associated with voluntary guided target detection. Interestingly, the largest voxel cluster (size: 132 voxels) was located within the precuneus/SPL bilaterally. The precuneus is a cortical structure located in the superior parietal cortex. It is one of the core structures of the DAN [4, 14] and associated with voluntary attentional switching [89], but also modulated by saliency [90]. Activation of the precuneus/SPL (e.g., BA 7) was also observed in the contrast comparing processing of deviants during active $>$ passive listening; again in the time P3 time window. In addition, P3 modulation was significantly larger for "Deviants" during active as compared to passive listening. Activation of the precuneus/SPL in the P3 time window during active listening may therefore indeed indicate the increase in attentional demands from passive to active, attentive and thus, voluntary and top-down controlled target detection.

Taken together, the SLORETA source localization analysis support the hypothesis that unattended (passive) as well as attended deviance and target detection elicit cortical activations in spatially distributed brain regions belonging to different brain networks including the VAN, DAN and SN.

\section{A neurophysiolological model of passive and active auditory deviance and target detection}

As illustrated in Fig. 4, based on the results of the present study a neurophysiological model of passive and active auditory deviance and target detection can be proposed that may act as a guide for future research. As shown in Fig. 4, this model illustrates that passive and active auditory deviance detection in the auditory oddball paradigm are associated with activation of brain regions belonging to at least two different brain networks: these include on the one hand auditory processing regions in the STG (e.g., BA 22) and the insula (BA 13) as key region of the VAN and SN involved in passive auditory deviance detection, and on the other hand, parietal and frontal brain regions as key regions of the VAN and particularly of the DAN involved in task-related auditory deviance and target detection [18, 91]. Activation of the STG and insula during passive listening is in close agreement with previously conducted neuroimaging studies combining fMRI with multi-channel EEG recordings during an auditory oddball paradigm and a passive listening task $[10,12]$. Results of these studies obtained from fMRI indicated comparable cortical activations in the right STG and the right superior temporal plane (BAs 41/42) and right anterior insula during passive listening of auditory deviant stimuli. Also, going beyond previous research, the present results suggest that during passive listening activation of the VAN and the SN in particular may occur at later stages of stimulus selection, i.e., when deviants in contrast to standards are selected for further processing and associated with the elicitation of a P3 component. Earlier processing stages associated with automatic deviance detection as reflected by N1/MMN modulation on the contrary seem to be related specifically with activation of sensory brain regions belonging to the VAN (superior temporal gyrus) as well as with visual cortical activation. Although brain regions in the visual cortex, such as the lateral occipital cortex are typically associated with the processing of visual information (e.g., visual objects) in visual spatial attention tasks [21-23], more recent functional imaging studies $[9,92]$ showed in line with our findings that specific regions in the visual cortex (such as the LOC or the lingual gyrus in the medial visual cortex) may be activated during the processing of salient acoustic stimuli [24] and as suggested by our study this may occur even or specifically if no task is at hand. Moreover, in contrast to passive listening, during active listening auditory processing may be fully taken over by the brain's attention networks including activation of brain regions belonging to the VAN during deviance detection in the N1/MMN time window and of the DAN during target detection in the P3 time window.

\section{Limitations and future outlook}

Although the results of the present study including the proposed model support a number of the hypotheses tested there are limitations that must be taken with caution. A major disadvantage of the present study may be the small sample size. However, effect sizes calculated for the $t$-tests reported under 3.2-3.5 revealed at least moderate effects (Cohen's $d \geq|0.6|$ ) resulting in a post hoc power estimation of at least 0.6. Nevertheless, due to the small 


\section{Auditory Oddball Paradigm}

\section{S S S SDSS SDSSDSS SDSSDSS S S S DS SD...}

a

$$
\text { passive listening condition }
$$

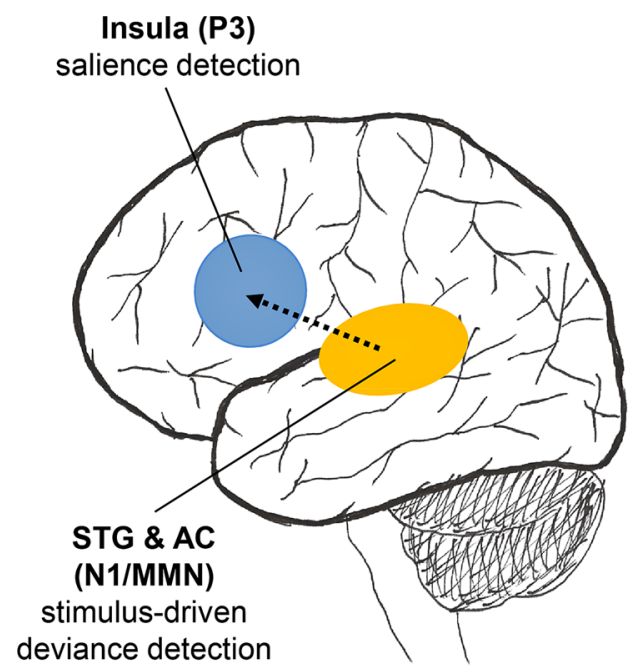

b

\section{active listening condition}

(voluntarily attending presented tones and pressing a key in response to deviants)

\author{
Prefronal Gyrus (P3) \\ task-driven \\ target detection
}

\section{SPL/Precuneus (P3) task-driven target detection}

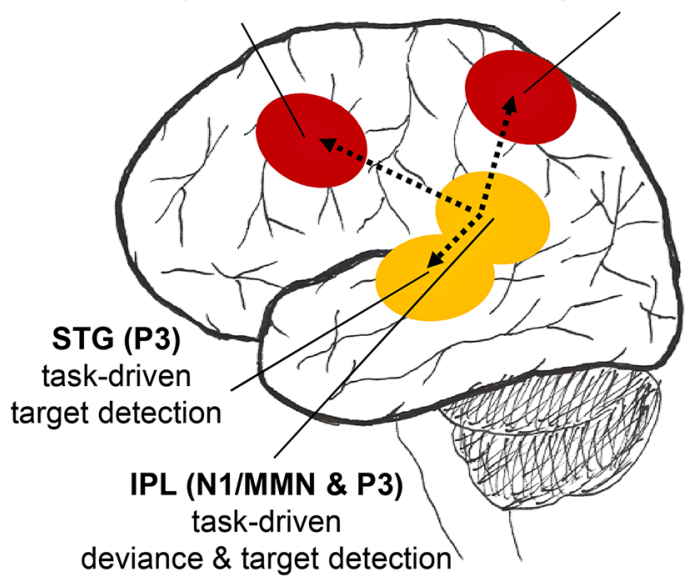

ventral attention network (VAN) dorsal attention network (DAN) salience network (SN)

Fig. 4 Overview of a proposed model based on the obtained ERP and SLORETA results (for an explanation, see "A neurophysiolological model of passive and active auditory deviance and target detection" section). AC auditory cortex, IPL inferior parietal lobule, MMN mismatch negativity, STG superior temporal gyrus, SPL superior parietal lobule

sample size the generalizability of the reported effects and the proposed model may be limited (see Fig. 4).

Another confound in the present study might be the temporal overlap of the $\mathrm{N} 1$ and MMN component. This phenomenon may be observed when the perceptual difference between "Deviants" and "Standards" is particularly prominent in the frequency domain [93]. Regarding the question which and how many brain networks may be activated during passive versus active deviance and target detection further research may unravel the functional connectivity between the hypothesized networks. The present results suggest that besides the VAN and DAN, the SN may indeed play an important role in auditory deviance and target detection during passive listening. In contrast to the VAN, the SN is believed to be involved in the detection of stimulus saliency, expectancy and automatic selection of an adaptive and suitable (behavioral) response [18]. The core structure of the $\mathrm{SN}$ consists of the dorsal part of the ACC (dACC; BAs 24/32/33), subcortical and limbic structures (e.g. amygdala), as well as both insulae bilaterally [91]. Given the high degree of functional and anatomical overlap between the SN and the VAN, some researchers see both networks as parts of the same system [94]. However, given that according to meta-analytic findings the VAN should be more active when stimuli are task-relevant which was confirmed in this study and the fact that, in this study activation of the insula was not found during active listening, the present results agree with the notion to conceptualize the $\mathrm{SN}$ and VAN as two distinct networks $[95,96]$ with distinct roles during auditory processing.

Yet, another restriction that needs to be mentioned is related to the mathematical algorithms implemented in sLORETA. These algorithms are mainly based on nonparametric voxel-by-voxel comparisons for why sLORETA results-like conventional fMRI results-should not be interpreted causally. To overcome some of these methodological limitations, the application of repetitive transcranial magnetic stimulation (rTMS) may offer a non-invasive and thus elegant way to selectively inhibit 
or facilitate cortical activity in superficial brain regions (e.g., IPL or SPL/precuneus) and even in deeper cortical structures such as the insula [97] by applying fast trains of electromagnetic pulses [98]. Hence, in future studies, rTMS would offer potential prove for the neurophysiological model derived from the present data regarding passive as well as active auditory deviance and target detection.

\section{Conclusion}

In summary, the present study investigated the temporal and spatial dynamics of auditory deviance and target detection in an auditory oddball paradigm by combining EEG-ERP and sLORETA methods. Despite abundant previous research investigating either the time course or the neural sources and brain structures of auditory processing in the auditory oddball paradigm the present study is one of the few studies so far that combined analysis of ERPs with sLORETA source imaging during passive and active listening conditions in a within subject design in an attempt to explore when and where in the brain auditory deviance and target detection takes place during passive and active listening conditions. The results of the present study as well as the neurophysiological model derived from the current findings may be tentative due to the small sample size but may bolster future studies validating the suggested temporal activation pattern in larger samples of participants.

\section{Authors' contributions}

Both authors contributed extensively to the work presented in this paper. Both authors made substantial contributions to the conception and design, the analysis and interpretation of the data. CJ and $\mathrm{CH}$ designed the experiment. CJ ran the experiment and collected the data. CJ analyzed the data with support of $\mathrm{CH}$. CJ and $\mathrm{CH}$ interpreted the data and $\mathrm{CJ}$ and $\mathrm{CH}$ wrote the manuscript. Both authors read and approved the final manuscript.

\section{Author details \\ ${ }^{1}$ University of Tuebingen, Tuebingen, Germany. ${ }^{2}$ Institute of Psychology and Education, Applied Emotion and Motivation Research, University of UIm, Ulm, Germany.}

\section{Acknowledgements}

We would like to thank Prof. Dr. Dr. Markus Raab for the opportunity to use the EEG laboratory located at the Institute of Psychology of the German Sport University Cologne, Germany.

\section{Competing interests}

The authors declare that they have no competing interests.

\section{Availability of data and materials}

The datasets used and/or analysed during the current study are available from the corresponding author on reasonable request.

\section{Consent for publication}

The manuscript does not include details, images, or videos relating to an individual person. All figures provided in this manuscript are created by the authors of this manuscript.

\section{Ethics approval and consent to participate}

The experiment (including EEG recordings) was conducted at the Institute of Psychology of the German Sport University Cologne, Germany. The experimental protocol complied with the Declaration of Helsinki and was approved by the local ethics committee of the German Sport University Cologne, Germany (https://www.dshs-koeln.de/institut-fuer-paedagogik-und-philosophie/ forschung/antraege-an-die-ethikkommission/). Participants gave written informed consent prior to the start of the experiment.

\section{Funding}

This study was supported by the German Research Foundation (DFG; HE5880$3 / 1)$, awarded to $\mathrm{CH}$

\section{Publisher's Note}

Springer Nature remains neutral with regard to jurisdictional claims in published maps and institutional affiliations.

Received: 7 February 2017 Accepted: 28 March 2018

Published online: 19 April 2018

\section{References}

1. Watkins S, Dalton P, Lavie N, Rees G. Brain mechanisms mediating auditory attentional capture in humans. Cereb Cortex. 2007;17:1694-700.

2. Halgren E, Squires NK, Wilson CL, Rohrbaugh JW, Babb TL, Crandall PH. Endogenous potentials generated in the human hippocampal formation and amygdala by infrequent events. Science. 1980;210:803 LP-805LP.

3. Bledowski C, Prvulovic D, Goebel R, Zanella FE, Linden DEJ. Attentional systems in target and distractor processing: a combined ERP and fMRI study. Neuroimage. 2004:22:530-40.

4. Kim H. Involvement of the dorsal and ventral attention networks in oddball stimulus processing: a meta-analysis. Hum Brain Mapp. 2014;35:2265-84

5. Bennington JY, Polich J. Comparison of P300 from passive and active tasks for auditory and visual stimuli. Int J Psychophysiol. 1999;34:171-7.

6. Gurtubay IG, Alegre M, Labarga A, Malanda A, Iriarte J, Artieda J. Gamma band activity in an auditory oddball paradigm studied with the wavelet transform. Clin Neurophysiol. 2001;112:1219-28.

7. Kennan RP, Horovitz SG, Maki A, Yamashita Y, Koizumi H, Gore JC. Simultaneous recording of event-related auditory oddball response using transcranial near infrared optical topography and surface EEG. Neuroimage. 2002;16:587-92.

8. Lee T-W, Yu YW-Y, Wu H-C, Chen T-J. Do resting brain dynamics predict oddball evoked-potential? BMC Neurosci. 2011;12:1-10.

9. Kiehl KA, Laurens KR, Duty TL, Forster BB, Liddle PF. Neural sources involved in auditory target detection and novelty processing: an eventrelated fMRI study. Psychophysiology. 2001;38:133-42.

10. Liebenthal E, Ellingson ML, Spanaki MV, Prieto TE, Ropella KM, Binder JR. Simultaneous ERP and fMRI of the auditory cortex in a passive oddball paradigm. Neuroimage. 2003;19:1395-404

11. Linden DEJ, Prvulovic D, Formisano E, Völlinger M, Zanella FE, Goebel R, et al. The functional neuroanatomy of target detection: an fMRI study of visual and auditory oddball tasks. Cereb Cortex. 1999;9:815-23.

12. Müller BW, Stude $P$, Nebel K, Wiese H, Ladd ME, Forsting M, et al. Sparse imaging of the auditory oddball task with functional MRI. NeuroReport. 2003;14:1597-601.

13. Opitz B, Mecklinger A, von Cramon DY, Kruggel F. Combining electrophysiological and hemodynamic measures of the auditory oddball. Psychophysiology. 1999;36:142-7.

14. Corbetta M, Shulman GL. Control of goal-directed and stimulus-driven attention in the brain. Nat Rev Neurosci. 2002;3:215-29.

15. Vossel S, Geng JJ, Fink GR. Dorsal and ventral attention systems: distinct neural circuits but collaborative roles. Neurosci. 2014:20:150-9.

16. Rossi AF, Pessoa L, Desimone R, Ungerleider LG. The prefrontal cortex and the executive control of attention. Exp Brain Res. 2009:192:489-97.

17. Palaniyappan L, Liddle PF. Does the salience network play a cardinal role in psychosis? An emerging hypothesis of insular dysfunction. J Psychiatry Neurosci. 2012;37:17-27. 
18. Menon V, Uddin LQ. Saliency, switching, attention and control: a network model of insula function. Brain Struct Funct. 2010;214:655-67.

19. Seeley WW, Menon V, Schatzberg AF, Keller J, Glover GH, Kenna H, et al. Dissociable intrinsic connectivity networks for salience processing and executive control. J Neurosci. 2007;27:2349-56.

20. Sridharan D, Levitin DJ, Menon V. A critical role for the right fronto-insular cortex in switching between central-executive and default-mode networks. Proc Natl Acad Sci. 2008;105:12569-74.

21. Grill-Spector K, Kushnir T, Edelman S, Avidan G, Itzchak Y, Malach R. Differential processing of objects under various viewing conditions in the human lateral occipital complex. Neuron. 1999;24:187-203.

22. Grill-Spector K, Kourtzi Z, Kanwisher N. The lateral occipital complex and its role in object recognition. Vis Res. 2001;41:1409-22.

23. Murray SO, Wojciulik E. Attention increases neural selectivity in the human lateral occipital complex. Nat Neurosci. 2004;7:70-4.

24. McDonald JJ, Störmer VS, Martinez A, Feng W, Hillyard SA. Salient sounds activate human visual cortex automatically. J Neurosci. 2013;33:9194-201.

25. Ji J, Porjesz B, Begleiter H, Chorlian D. P300: the similarities and differences in the scalp distribution of visual and auditory modality. Brain Topogr Kluwer Acad Publ Plenum Publ. 1999;11:315-27.

26. Kemner C, Verbaten MN, Cuperus JM, Camfferman G, van Engeland H. Auditory event-related brain potentials in autistic children and three different control groups. Biol Psychiatry. 1995;38:150-65.

27. Anderer P, Pascual-Marqui RD, Semlitsch HV, Saletu B. Differential effects of normal aging on sources of standard N1, target N1 and target P300 auditory event-related brain potentials revealed by low resolution electromagnetic tomography (LORETA). Electroencephalogr Clin Neurophysiol Potentials Sect. 1998;108:160-74.

28. Grimm S, Escera C. Auditory deviance detection revisited: evidence for a hierarchical novelty system. Int J Psychophysiol. 2012;85:88-92.

29. Polich J. Updating P300: an integrative theory of P3a and P3b. Clin Neurophysiol. 2007;118:2128-48.

30. Polich J, Kok A. Cognitive and biological determinants of P300: an integrative review. Biol Psychol. 1995;41:103-46.

31. Squires NK, Squires KC, Hillyard SA. Two varieties of long-latency positive waves evoked by unpredictable auditory stimuli in man. Electroencephalogr Clin Neurophysiol. 1975;38:387-401.

32. Luck SJ. An introduction to the event-related potential technique. Cambrigde: MIT Press; 2005.

33. Näätänen R, Paavilainen $P$, Rinne T, Alho K. The mismatch negativity (MMN) in basic research of central auditory processing: a review. Clin Neurophysiol. 2007;118:2544-90.

34. Garrido MI, Kilner JM, Stephan KE, Friston KJ. The mismatch negativity: a review of underlying mechanisms. Clin Neurophysiol Off J Int Fed Clin Neurophysiol. 2009;120:453-63.

35. Duncan CC, Barry RJ, Connolly JF, Fischer C, Michie PT, Näätänen R, et al. Event-related potentials in clinical research: guidelines for eliciting, recording, and quantifying mismatch negativity, P300, and N400. Clin Neurophysiol. 2009;120:1883-908.

36. Campbell T, Winkler I, Kujala T. N1 and the mismatch negativity are spatiotemporally distinct ERP components: disruption of immediate memory by auditory distraction can be related to N1. Psychophysiology. 2007:44:530-40.

37. Näätänen R, Picton T. The N1 wave of the human electric and magnetic response to sound: a review and an analysis of the component structure. Psychophysiology. 1987;24:375-425.

38. Näätänen R, Alho K. Mismatch negativity—a unique measure of sensory processing in audition. Int J Neurosci. 1995;80:317-37.

39. Escera C, Alho K, Schröger E, Winkler I. Involuntary attention and distractibility as evaluated with event-related brain potentials. Audiol Neuro Otol. 2000:5:151-66.

40. Escera C, Alho K, Winkler I, Näätänen R. Neural mechanisms of involuntary attention to acoustic novelty and change. J Cogn Neurosci. 1998;10:590-604.

41. Patel SH, Azzam PN. Characterization of N200 and P300: selected studies of the event-related potential. Int J Med Sci. 2005;2(4):147-54

42. Picton TW. The P300 wave of the human event-related potential. J Clin Neurophysiol. 1992;9:456-79.

43. Debener S, Kranczioch C, Herrmann CS, Engel AK. Auditory novelty oddball allows reliable distinction of top-down and bottom-up processes of attention. Int J Psychophysiol. 2002;46:77-84.
44. Kok A. On the utility of P3 amplitude as a measure of processing capacity. Psychophysiology. 2001;38:557-77.

45. Donchin E, Coles M. Is the P300 component a manifestation of context updating? Behav Brain Sci. 1988;11:357-74.

46. Birn RM, Cox RW, Bandettini PA. Detection versus estimation in eventrelated fMRI: choosing the optimal stimulus timing. Neuroimage. 2002;15:252-64.

47. Pascual-Marqui RD. Review of methods for solving the EEG inverse problem. Int J Bioelectromagn. 1999;1:75-86.

48. Vitacco D, Brandeis D, Pascual-Marqui R, Martin E. Correspondence of event-related potential tomography and functional magnetic resonance imaging during language processing. Hum Brain Mapp. 2002;17:4-12.

49. Pascual-Marqui RD, Michel CM, Lehmann D. Low resolution electromagnetic tomography: a new method for localizing electrical activity in the brain. Int J Psychophysiol. 1994;18:49-65.

50. Mulert C, Jäger L, Schmitt R, Bussfeld P, Pogarell O, Möller H-J, et al. Integration of fMRI and simultaneous EEG: towards a comprehensive understanding of localization and time-course of brain activity in target detection. Neuroimage. 2004;22:83-94.

51. Mulert C, Pogarell O, Juckel G, Rujescu D, Giegling I, Rupp D, et al. The neural basis of the P300 potential. Eur Arch Psychiatry Clin Neurosci. 2004:254:190-8.

52. Volpe U, Mucci A, Bucci P, Merlotti E, Galderisi S, Maj M. The cortical generators of P3a and P3b: a LORETA study. Brain Res Bull. 2007;73:220-30.

53. Justen $C$, Herbert C. Snap your fingers! An ERP/sLORETA study investigating implicit processing of self- vs. other-related movement sounds using the passive oddball paradigm. Front Hum Neurosci. 2016;10:465.

54. Williams LM, Simms E, Clark CR, Paul RH, Rowe D, Gordon E. The testretest reliability of a standardized neurocognitive and neurophysiological test battery:"Neuromarker". Int J Neurosci. 2005;115:1605-30.

55. Millisecond: Auditory Oddball Task [Internet]. 2017 [cited 2017 Jan 28]. http://www.millisecond.com/download/library/Oddball/.

56. Jurcak V, Tsuzuki D, Dan I. 10/20, 10/10, and 10/5 systems revisited: their validity as relative head-surface-based positioning systems. Neuroimage. 2007:34:1600-11.

57. Zanow F, Knösche TR. ASA_advanced source analysis of continuous and event-related EEG/MEG signals. Brain Topogr. 2004;16:287-90.

58. Ille N, Berg P, Scherg M. Artifact correction of the ongoing EEG ssing spatial filters based on artifact and brain signal topographies. J Clin Neurophysiol. 2002;19:113-24.

59. Delorme A, Makeig S. EEGLAB: an open source toolbox for analysis of single-trial EEG dynamics including independent component analysis. $J$ Neurosci Methods. 2004;134:9-21.

60. Delorme A. Statistical methods. In: Webster JG, editor. Encyclopedia of medical devices and instrumentation. Hoboken: Wiley; 2004. p. 240-56.

61. Benjamini Y, Hochberg Y. Controlling the false discovery rate: a practical and powerful approach to multiple testing. J R Stat Soc Ser B. 1995;1:289-300.

62. Lage-Castellanos A, Martínez-Montes E, Hernández-Cabrera JA, Galán L. False discovery rate and permutation test: an evaluation in ERP data analysis. Stat Med. 2010;29:63-74.

63. Groppe DM, Urbach TP, Kutas M. Mass univariate analysis of event-related brain potentials/fields I: a critical tutorial review. Psychophysiology. 2011:48:1711-25

64. Groppe DM, Urbach TP, Kutas M. Mass univariate analysis of eventrelated brain potentials/fields II: simulation studies. Psychophysiology. 2011:48:1726-37.

65. Mass Univariate ERP Toolbox [Internet]. 2017 [cited 2017 Jan 28]. http:// openwetware.org/wiki/Mass_Univariate_ERP_Toolbox.

66. Bullmore ET, Suckling J, Overmeyer S, Rabe-Hesketh S, Taylor E, Brammer MJ. Global, voxel, and cluster tests, by theory and permutation, for a difference between two groups of structural MR images of the brain. IEEE Trans Med Imaging. 1999;18:32-42.

67. Manly BFJ. Randomization, bootstrap and Monte Carlo methods in biology texts in statistical science. 2nd ed. London: Chapman \& Hall/CRC; 1997.

68. LORETA—Low Resolution Electromagnetic Tomography [Internet]. 2017 [cited 2017 Jan 28]. http://www.uzh.ch/keyinst/loreta.htm.

69. Fuchs M, Kastner J, Wagner M, Hawes S, Ebersole JS. A standardized boundary element method volume conductor model. Clin Neurophysiol. 2002:113:702-12. 
70. Mazziotta J, Toga A, Evans A, Fox P, Lancaster J, Zilles K, et al. A probabilistic atlas and reference system for the human brain: International Consortium for Brain Mapping (ICBM). Philos Trans R Soc Lond B Biol Sci R. Soc. 2001;356:1293-322.

71. Lancaster JL, Woldorff MG, Parsons LM, Liotti M, Freitas CS, Rainey L, et al. Automated Talairach Atlas labels for functional brain mapping. Hum Brain Mapp. 2000;10:120-31.

72. Talairach J, Tournoux P.: Co-planar stereotaxic atlas of the human brain. Stuttgart; New York: G. Thieme; 1988.

73. Oostenveld R, Praamstra P. The five percent electrode system for high-resolution EEG and ERP measurements. Clin Neurophysiol. 2001;112:713-9.

74. Brett M, Johnsrude IS, Owen AM. The problem of functional localization in the human brain. Nat Rev Neurosci. 2002;3:243-9.

75. Nichols TE, Holmes AP. Nonparametric permutation tests for functional neuroimaging: a primer with examples. Hum Brain Mapp. 2002;15:1-25.

76. Silva L, Amitai Y, Connors B. Intrinsic oscillations of neocortex generated by layer 5 pyramidal neurons. Science. 1991;251:433-5.

77. Haalman I, Vaadia E. Dynamics of neuronal interactions: relation to behavior, firing rates, and distance between neurons. Hum Brain Mapp. 1997:5:249-53.

78. Friston KJ. Statistical parametric mapping and other analysis of functional imaging data. In: Toga AW, Mazziotta JC, editors. Brain Mapp. 2nd. ed. Amsterdam; Boston: Academic Press; 2002. p. 363-85.

79. Brodmann K, Gary LJ. Brodmann's localization in the cerebral cortex. New York: Springer; 2006.

80. Koelsch S. Brain and Music. 1st ed. Chichester, West Sussex; Hoboken, NJ: Wiley-Blackwell: John Wiley \& Sons, Inc; 2012.

81. Godey B, Schwartz D, de Graaf J, Chauvel P, Liégeois-Chauvel C. Neuromagnetic source localization of auditory evoked fields and intracerebral evoked potentials: a comparison of data in the same patients. Clin Neurophysiol. 2001;112:1850-9.

82. Zouridakis G, Simos PG, Papanicolaou AC. Multiple bilaterally asymmetric cortical sources account for the auditory N1 m component. Brain Topogr. 1998;10:183-9.

83. McEvoy L, Hari R, Imada T, Sams M. Human auditory cortical mechanisms of sound lateralization: II. Interaural time differences at sound onset. Hear Res. 1993;67:98-109.

84. Näätänen R, Sams M, Alho K, Paavilainen P, Reinikainen K, Sokolov EN Frequency and location specificify of the human vertex N1 wave. Electroencephalogr Clin Neurophysiol. 1988;69:523-31.

85. Jääskeläinen IP, Ahveninen J, Bonmassar G, Dale AM, IImoniemi RJ, Levänen $\mathrm{S}$, et al. Human posterior auditory cortex gates novel sounds to consciousness. Proc Natl Acad Sci USA. 2004;101:6809-14.
86. Singh-Curry $V$, Husain $M$. The functional role of the inferior parietal lobe in the dorsal and ventral stream dichotomy. Neuropsychologia. 2009:47:1434-48.

87. Bamiou D-E, Musiek FE, Luxon LM. The insula (Island of Reil) and its role in auditory processing. Brain Res Rev. 2003;42:143-54.

88. Müller BW, Achenbach C, Oades RD, Bender S, Schall U. Modulation of mismatch negativity by stimulus deviance and modality of attention. Neuroreport. 2002;13

89. Le TH, Pardo JV, Hu X.4T-fMRI study of nonspatial shifting of selective attention: cerebellar and parietal contributions. J Neurophysiol. 1998;79:1535-48.

90. Indovina I, Macaluso E. Dissociation of stimulus relevance and saliency factors during shifts of visuospatial attention. Cereb Cortex. 2007;17:1701-11.

91. Uddin LQ. Salience processing and insular cortical function and dysfunction. Nat Rev Neurosci. 2014;16:55-61.

92. Goldman RI, Wei C-Y, Philiastides MG, Gerson AD, Friedman D, Brown TR, et al. Single-trial discrimination for integrating simultaneous EEG and fMRI: identifying cortical areas contributing to trial-to-trial variability in the auditory oddball task. Neuroimage. 2009;47:136-47.

93. Tiitinen H, May P, Reinikainen K, Näätänen R. Attentive novelty detection in humans is governed by pre-attentive sensory memory. Nature. 1994;372:90-2.

94. Kucyi A, Hodaie M, Davis KD. Lateralization in intrinsic functional connectivity of the temporoparietal junction with salience- and attention-related brain networks. J Neurophysiol. 2012;108:3382-92.

95. Cole MW, Reynolds JR, Power JD, Repovs G, Anticevic A, Braver TS. Multi-task connectivity reveals flexible hubs for adaptive task control. Nat Neurosci. 2013:16:1348-55.

96. Power JD, Cohen AL, Nelson SM, Wig GS, Barnes KA, Church JA, et al. Functional network organization of the human brain. Neuron. 2011;72:665-78.

97. Ciampi de Andrade D, Galhardoni R, Pinto LF, Lancelotti R, Rosi J Jr, Marcolin MA, et al. Into the Island: a new technique of non-invasive cortical stimulation of the insula. Neurophysiol Clin Neurophysiol. 2012;42:363-8.

98. Walsh V, Cowey A. Transcranial magnetic stimulation and cognitive neuroscience. Nat Rev Neurosci. 2000;1:73-9.
Ready to submit your research? Choose BMC and benefit from:

- fast, convenient online submission

- thorough peer review by experienced researchers in your field

- rapid publication on acceptance

- support for research data, including large and complex data types

- gold Open Access which fosters wider collaboration and increased citations

- maximum visibility for your research: over 100M website views per year

At BMC, research is always in progress.

Learn more biomedcentral.com/submissions 